PROCEEDINGS OF THE

AMERICAN MATHEMATICAL SOCIETY

Volume 131, Number 4, Pages 1063-1070

S 0002-9939(02)06725-4

Article electronically published on July 26, 2002

\title{
AVERAGE VALUES OF SYMMETRIC SQUARE $L$-FUNCTIONS AT THE EDGE OF THE CRITICAL STRIP
}

\author{
J. WU
}

(Communicated by Dennis A. Hejhal)

AbStract. Let $\mathcal{B}_{2}^{*}(N)$ be the set of all normalized newforms of weight 2 and level $N$, and let $L\left(\operatorname{sym}^{2} f, 1\right)$ be the symmetric square $L$-function associated to $f \in \mathcal{B}_{2}^{*}(N)$. If $N$ is a prime, then there is a positive constant $B$ such that

$$
\sum_{f \in \mathcal{B}_{2}^{*}(N)} L\left(1, \operatorname{sym}^{2} f\right)=\frac{\pi^{4}}{432} N+O\left(N^{27 / 28}(\log N)^{B}\right) .
$$

This improves a recent result of Akbary, which requires 45/46 in place of $27 / 28$.

\section{§1. INTRODUCTION}

Let $S_{2}(N)$ be the set of all cusp forms of weight 2 for the full modular group $\Gamma_{0}(N)$ with trivial character. This is a finite-dimensional Hilbert space with respect to the Petersson inner product defined by

$$
\langle f, g\rangle:=\int_{\Gamma_{0}(N) \backslash \mathbb{H}} f(z) \overline{g(z)} \mathrm{d} x \mathrm{~d} y,
$$

where $\mathbb{H}$ is the upper half-plane. Let $\mathcal{B}_{2}^{*}(N)$ be the set of all normalized newforms in $S_{2}(N)$. Every $f \in \mathcal{B}_{2}^{*}(N)$ has the Fourier expansion of type

$$
f(z)=\sum_{n=1}^{\infty} \lambda_{f}(n) n^{1 / 2} e(n z),
$$

where $e(z):=e^{2 \pi i z}, \lambda_{f}(1)=1$ and $\lambda_{f}(n) \in \mathbb{R}$.

The symmetric square $L$-function associated to $f \in \mathcal{B}_{2}^{*}(N)$ is defined by

$$
L\left(s, \operatorname{sym}^{2} f\right):=\zeta_{N}(2 s) \sum_{n=1}^{\infty} \frac{\lambda_{f}\left(n^{2}\right)}{n^{s}} \quad(\Re e s>1),
$$

where

$$
\zeta_{N}(s):=\prod_{p \nmid N}\left(1-1 / p^{s}\right)^{-1} .
$$

Define

$$
L_{\infty}\left(s, \operatorname{sym}^{2} f\right):=\left(\frac{N}{\pi^{3 / 2}}\right)^{s} \Gamma\left(\frac{s+1}{2}\right)^{2} \Gamma\left(\frac{s+2}{2}\right) .
$$

Received by the editors November 12, 2001.

2000 Mathematics Subject Classification. Primary 11F67.

(C)2002 American Mathematical Society 
Then $\Lambda\left(s, \operatorname{sym}^{2} f\right):=L_{\infty}\left(s, \operatorname{sym}^{2} f\right) L\left(s, \operatorname{sym}^{2} f\right)$ is an entire function and satisfies the functional equation

$$
\Lambda\left(s, \operatorname{sym}^{2} f\right)=\Lambda\left(1-s, \operatorname{sym}^{2} f\right) .
$$

Similar to the class number formula of Dirichlet, the value of $L\left(s, \operatorname{sym}^{2} f\right)$ at the edge of the critical strip (in this case $s=1$ ) is of interest. When $N$ is square free, one can show that (cf. [3], Lemma 2.5)

$$
L\left(1, \operatorname{sym}^{2} f\right)=\frac{8 \pi^{3}\langle f, f\rangle}{N} .
$$

Therefore to study the average values of the Petersson inner product when $f$ varies in $\mathcal{B}_{2}^{*}(N)$, it is enough to find an asymptotic formula for the average values of $L\left(1, \operatorname{sym}^{2} f\right)$.

The mean values of $L\left(1, \operatorname{sym}^{2} f\right)$ were first investigated by R. Murty [6]. He proved that if we assume that $L\left(\frac{1}{2}+i \tau, \operatorname{sym}^{2} f\right) \ll(N(|\tau|+1))^{\theta}$ for some $\theta>0$, then for prime $N$ we have

$$
\sum_{f \in \mathcal{B}_{2}^{*}(N)} L\left(1, \operatorname{sym}^{2} f\right)=\frac{\pi^{4}}{432} N+O\left(N^{7 / 10+4 \theta / 5} \mathcal{L}^{3}\right),
$$

where here and in the sequel we systematically write $\mathcal{L}:=\log N$. According to ([5], page 336]), the value $\theta=3 / 4$ is actually permissible. Thus the estimate (4) is only a conditional asymptotic formula. Very recently Akbary [1 has obtained an unconditional result: If $N$ is a prime, there is a positive constant $B$ such that

$$
\sum_{f \in \mathcal{B}_{2}^{*}(N)} L\left(1, \operatorname{sym}^{2} f\right)=\frac{\pi^{4}}{432} N+O\left(N^{45 / 46} \mathcal{L}^{B}\right) .
$$

In order to prove this asymptotic formula, he has combined the method of Duke [2] and that of Kowalski and Michel [4].

In this paper, we shall propose a better result.

Theorem. Let $N$ be a prime. Then there is a positive constant $B$ such that

$$
\sum_{f \in \mathcal{B}_{2}^{*}(N)} L\left(1, \operatorname{sym}^{2} f\right)=\frac{\pi^{4}}{432} N+O\left(N^{27 / 28} \mathcal{L}^{B}\right) .
$$

For comparison, we have $\frac{45}{46} \approx 0.978$ and $\frac{27}{28} \approx 0.964$. Our improvement comes from two observations. First we find that only applying the method of 4 is more effective than the combined method of [2] and [4]. Secondly we give a straightforward improvement of the method of [4], which allows us to make use of a wider range of parameters (see Lemma 2 below). Without the second remark, we only have $29 / 30$ in place of $27 / 28$.

\section{$\S 2$. Some Lemmas}

As in [4], we define

$$
\omega_{f}(x, y):=\sum_{x<d^{2} m \leq y} \frac{\varepsilon_{N}(d) \lambda_{f}\left(m^{2}\right)}{d^{2} m},
$$


where

$$
\varepsilon_{N}(d):= \begin{cases}1 & \text { if }(d, N)=1 \\ 0 & \text { otherwise. }\end{cases}
$$

The next lemma is essentially Lemma 6 of [4, but the second term here is sharpened. This improvement does not need to add any new idea. In fact their proof can yield such a result. For completeness, we reproduce their proof here with some minor modifications.

Lemma 1. Let $r \geq 1$ be a fixed integer and $x, y, z \geq 2$ with $y>x$. Then there exist a real number $M \in\left[x^{r} z^{-1}, y^{r} z\right]$ and real numbers $c(m)$ such that

$$
\sum_{f \in \mathcal{B}_{2}^{*}(N)} \omega_{f}(x, y)^{2 r} \ll\left(\sum_{f \in \mathcal{B}_{2}^{*}(N)}\left|\sum_{m \sim M} \lambda_{f}\left(m^{2}\right) \frac{c(m)}{m}\right|^{2}+N z^{-1}\right)\{\log (N y z)\}^{B_{1}}
$$

and

$$
|c(m)| \ll\{\tau(m) \log z\}^{B_{2}}
$$

where the $B_{i}=B_{i}(r)$ are some positive constants and $\tau(m)$ is the usual divisor function.

Proof. By Lemmas 4 and 5 of [4, we have

$$
\omega_{f}(x, y)^{r}=\sum_{\substack{x^{r}<m n \leq y^{r} \\ n \leq z}} \lambda_{f}\left(m^{2}\right) \frac{c(m, n)}{m n}+O\left(z^{-1 / 2}\{\log (N y z)\}^{B_{3}}\right),
$$

where $c(m, n)$ is defined as in Lemma 4 of $\left[4\right.$ and $B_{3}=B_{3}(r) \geq 1$ is a constant. Introducing

$$
c(m):=\sum_{x^{r} m^{-1}<n \leq z} \frac{c(m, n)}{n},
$$

the preceding estimate can be written as

$$
\omega_{f}(x, y)^{r}=\sum_{x^{r} z^{-1}<m \leq y^{r} z} \lambda_{f}\left(m^{2}\right) \frac{c(m)}{m}+O\left(z^{-1 / 2}\{\log (N y z)\}^{B_{3}}\right) .
$$

After a classic dyadic splitting, we find for some $M \in\left[x^{r} z^{-1}, y^{r} z\right]$,

$$
\omega_{f}(x, y)^{r} \ll\left(\left|\sum_{m \sim M} \lambda_{f}\left(m^{2}\right) \frac{c(m)}{m}\right|+z^{-1 / 2}\right)\{\log (N y z)\}^{B_{3}} .
$$

By using the inequality $(a+b)^{2} \leq 2\left(a^{2}+b^{2}\right)(a, b \in \mathbb{R})$ and the fact that $\left|\mathcal{B}_{2}^{*}(N)\right| \asymp$ $N$, we deduce the inequality (6) with $B_{1}=2 B_{3}$.

It remains to prove the upper bound for $c(m)$. Since $|c(m, n)| \leq \tau(m n)^{B_{4}}$ for some positive constant $B_{4}=B_{4}(r)$ (4], Lemma 4), we have

$$
|c(m)| \leq \sum_{n \leq z} \frac{\tau(m n)^{B_{4}}}{n} \leq \tau(m)^{B_{4}} \sum_{n \leq z} \frac{\tau(n)^{B_{4}}}{n} \ll\{\tau(m) \log z\}^{B_{2}} .
$$

This completes the proof of Lemma 1.

With the help of Lemma 1, we can improve Lemma 3 of [4]. The next lemma enlarges the admissible range of parameters in Lemma 3 of [4]. This is one of two keys in the proof of our theorem. 
Lemma 2. Let $r \geq 1$ be a fixed integer, $x^{r} \geq N^{10}$ and $y>x$ with $\log y \ll \mathcal{L}$. There exists a positive constant $B_{5}=B_{5}(r)$ such that

$$
\sum_{f \in \mathcal{B}_{2}^{*}(N)} \omega_{f}(x, y)^{2 r} \ll \mathcal{L}^{B_{5}} .
$$

Proof. We take $z=N$ in Lemma 1 (instead of $z=N^{2}$ as in 44). Then the assumption $x^{r} \geq N^{10}$ implies that $M \geq N^{9}$, and we may appeal to the mean value estimate of Corollary 1 of [4] (with $a(m)=c(m) / m \mathcal{L}^{B_{2}}$ ) to bound the double sums on the right-hand side of (6). This completes the proof of Lemma 2.

The following lemma is an improved version of Proposition 1 of [1], which is feasible for greater range of parameters.

Lemma 3. Let $r \geq 1$ be a fixed integer and $x^{r} \geq N^{10}$. There exists a positive constant $B_{6}=B_{6}(r)$ such that

$$
\sum_{f \in \mathcal{B}_{2}^{*}(N)} \lambda_{f}(n)=\frac{N}{12 \sqrt{n}} \delta_{n=\square}+O\left(N^{1-1 / 2 r} \tau(n) \mathcal{L}^{B_{6}}+N^{-1 / 2} x \sqrt{n} \tau(n)\right),
$$

where $\delta_{n=\square}=1$ if $n$ is a square and $\delta_{n=\square}=0$ otherwise.

Proof. Without lost of generality, we can assume that $x \leq N^{3 / 2}$ since $\left|\lambda_{f}(n)\right| \leq \tau(n)$ (Deligne's bound) and $\left|\mathcal{B}_{2}^{*}(N)\right| \asymp N$.

Let $y \in(x, \infty) \backslash \mathbb{N}$ with $\log y \ll \mathcal{L}$. By the Perron formula, we can write

$$
\sum_{d^{2} m<y} \frac{\varepsilon_{N}(d) \lambda_{f}\left(m^{2}\right)}{d^{2} m}=\frac{1}{2 \pi i} \int_{(1)} L\left(\operatorname{sym}^{2} f, s+1\right) \frac{y^{s}}{s} \mathrm{~d} s
$$

where $(\sigma):=\{\sigma+i \tau:-\infty<\tau<\infty\}$. Upon moving the line of integration from (1) to $(-2)$ and using the functional equation (2), the theorem of residues allows us to deduce

$$
\begin{aligned}
& \sum_{d^{2} m<y} \frac{\varepsilon_{N}(d) \lambda_{f}\left(m^{2}\right)}{d^{2} m} \\
& \quad=L\left(1, \operatorname{sym}^{2} f\right)+\frac{1}{2 \pi i A} \int_{(-2)} L\left(-s, \operatorname{sym}^{2} f\right) \frac{\Gamma\left(\frac{1-s}{2}\right)^{2} \Gamma\left(\frac{2-s}{2}\right)}{\Gamma\left(\frac{2+s}{2}\right)^{2} \Gamma\left(\frac{s+3}{2}\right)}\left(\frac{y}{A^{2}}\right)^{s} \frac{\mathrm{d} s}{s},
\end{aligned}
$$

where $A=N / \pi^{3 / 2}$. Since $L\left(\operatorname{sym}^{2} f, s\right)$ is absolutely convergent for $\Re e s>1$, it is easy to see that the last integral is $\ll N^{3} y^{-2}$. Hence we obtain

$$
L\left(1, \operatorname{sym}^{2} f\right)=\sum_{d^{2} m<y} \frac{\varepsilon_{N}(d) \lambda_{f}\left(m^{2}\right)}{d^{2} m}+O\left(N^{3} y^{-2}\right) .
$$

As in [4], we write

$$
L\left(1, \operatorname{sym}^{2} f\right)=\sum_{d^{2} m \leq x} \frac{\varepsilon_{N}(d) \lambda_{f}\left(m^{2}\right)}{d^{2} m}+\omega_{f}(x, y)+O\left(N^{3} y^{-2}\right),
$$

where $\omega_{f}(x, y)$ is defined by $(5)$. 
In view of (3), we can write

$$
\begin{aligned}
\sum_{f \in \mathcal{B}_{2}^{*}(N)} \lambda_{f}(n) & =\sum_{f \in \mathcal{B}_{2}^{*}(N)} \frac{L\left(1, \mathrm{sym}^{2} f\right)}{L\left(1, \mathrm{sym}^{2} f\right)} \lambda_{f}(n) \\
& =\frac{N}{2 \pi^{2}} \sum_{f \in \mathcal{B}_{2}^{*}(N)} \frac{\lambda_{f}(n)}{4 \pi\langle f, f\rangle} L\left(1, \mathrm{sym}^{2} f\right)
\end{aligned}
$$

By using (7), we deduce

$$
\sum_{f \in \mathcal{B}_{2}^{*}(N)} \lambda_{f}(n)=M+R_{1}+O\left(R_{2}\right)
$$

where

$$
\begin{aligned}
M & :=\frac{N}{2 \pi^{2}} \sum_{f \in \mathcal{B}_{2}^{*}(N)} \frac{\lambda_{f}(n)}{4 \pi\langle f, f\rangle} \sum_{d^{2} m \leq x} \frac{\varepsilon_{N}(d) \lambda_{f}\left(m^{2}\right)}{d^{2} m}, \\
R_{1} & :=\frac{N}{2 \pi^{2}} \sum_{f \in \mathcal{B}_{2}^{*}(N)} \frac{\lambda_{f}(n)}{4 \pi\langle f, f\rangle} \omega_{f}(x, y), \\
R_{2} & :=\frac{N^{4}}{y^{2}} \sum_{f \in \mathcal{B}_{2}^{*}(N)} \frac{\left|\lambda_{f}(n)\right|}{\langle f, f\rangle} .
\end{aligned}
$$

In order to evaluate $M$, we recall the following well-known estimate ([6], Proposition 1):

$$
\sum_{f \in \mathcal{B}_{2}^{*}(N)} \frac{\lambda_{f}(m) \lambda_{f}(n)}{4 \pi\langle f, f\rangle}=\delta_{m, n}+O\left(N^{-3 / 2}(m, n)^{1 / 2}(m n)^{1 / 2}\right),
$$

where $\delta_{m, n}$ is the diagonal Kronecker symbol. It follows that

$$
\begin{aligned}
M & =\frac{N}{2 \pi^{2}} \sum_{d^{2} m \leq x} \frac{\varepsilon_{N}(d)}{d^{2} m} \sum_{f \in \mathcal{B}_{2}^{*}(N)} \frac{\lambda_{f}\left(m^{2}\right) \lambda_{f}(n)}{4 \pi\langle f, f\rangle} \\
& =\frac{N}{2 \pi^{2}} \sum_{d^{2} m \leq x} \frac{\varepsilon_{N}(d)}{d^{2} m} \delta_{m^{2}, n}+O\left(R_{3}\right),
\end{aligned}
$$

where

$$
\begin{aligned}
R_{3} & =\left(\frac{n}{N}\right)^{1 / 2} \sum_{d^{2} m \leq x} \frac{\left(m^{2}, n\right)^{1 / 2}}{d^{2}} \\
& =\left(\frac{n}{N}\right)^{1 / 2} \sum_{\ell \mid n} \ell^{1 / 2} \sum_{m \leq x, \ell \mid m^{2}} \sum_{d^{2} \leq x / m} \frac{1}{d^{2}} .
\end{aligned}
$$


Now $\ell$ can be written uniquely as $\ell=\ell_{1} \ell_{2}^{2}$ with $\ell_{1}$ square free. Then we have $\ell \mid m^{2}$ if and only if $\ell_{1} \ell_{2} \mid m$. Therefore we have $m=\ell_{1} \ell_{2} m^{\prime}$ and

$$
\begin{aligned}
R_{3} & \ll\left(\frac{n}{N}\right)^{1 / 2} \sum_{\ell \mid n} \ell^{1 / 2} \sum_{m \leq x, \ell \mid m^{2}} 1 \\
& \ll\left(\frac{n}{N}\right)^{1 / 2} \sum_{\ell \mid n} \ell^{1 / 2} \sum_{m^{\prime} \leq x / \ell_{1} \ell_{2}} 1 \\
& \ll x\left(\frac{n}{N}\right)^{1 / 2} \sum_{\ell \mid n} \frac{\ell^{1 / 2}}{\ell_{1} \ell_{2}} \\
& \ll N^{-1 / 2} x \sqrt{n} \tau(n)
\end{aligned}
$$

since $\left(\ell_{1} \ell_{2}\right)^{2} \geq \ell_{1} \ell_{2}^{2}=\ell$.

If $n=k^{2}$, then

$$
\begin{aligned}
\sum_{d^{2} m \leq x} \frac{\varepsilon_{N}(d)}{d^{2} m} \delta_{m^{2}, k^{2}} & =\frac{1}{k} \sum_{d \leq(x / k)^{1 / 2}} \frac{\varepsilon_{N}(d)}{d^{2}} \\
& =\frac{1}{k}\left(\zeta_{N}(2)-\sum_{d>(x / k)^{1 / 2}} \frac{\varepsilon_{N}(d)}{d^{2}}\right) \\
& =\frac{\pi^{2}}{6 \sqrt{n}}+O\left(x^{-1 / 2} n^{-1 / 4}+N^{-2} n^{-1 / 2}\right)
\end{aligned}
$$

Thus we can write, for any positive integer $n$,

$$
\sum_{d^{2} m \leq x} \frac{\varepsilon_{N}(d)}{d^{2} m} \delta_{m^{2}, n}=\left\{\frac{\pi^{2}}{6 \sqrt{n}}+O\left(x^{-1 / 2} n^{-1 / 4}+N^{-2} n^{-1 / 2}\right)\right\} \delta_{n=\square}
$$

Combining these estimates, we find that

$$
M=\frac{N}{12 \sqrt{n}} \delta_{n=\square}+O\left(N^{-1 / 2} x \sqrt{n} \tau(n)+N x^{-1 / 2} n^{-1 / 4} \delta_{n=\square}+n^{-1 / 2} \delta_{n=\square}\right) .
$$

By using Deligne's inequality $\left|\lambda_{f}(n)\right| \leq \tau(n)$ and (9) with $m=n=1$, we have

$$
R_{2} \ll N^{4} y^{-2} \tau(n)
$$

Let $r^{\prime}>1$ such that $1 / r^{\prime}+1 / 2 r=1$. By using the inequality $\left|\lambda_{f}(n)\right| \leq \tau(n)$, the estimate $\langle f, f\rangle \gg N / \mathcal{L}$ (see [2], Proposition 4), the Hölder inequality and Lemma 2 , we deduce that there exists a positive constant $B_{6}=B_{6}(r)$ such that

$$
\begin{aligned}
R_{1} & \ll \tau(n) \mathcal{L}\left|\mathcal{B}_{2}^{*}(N)\right|^{1 / r^{\prime}}\left(\sum_{f \in \mathcal{B}_{2}^{*}(N)} \omega_{f}(x, y)^{2 r}\right)^{1 / 2 r} \\
& \ll N^{1-1 / 2 r} \tau(n) \mathcal{L}^{B_{6}} .
\end{aligned}
$$

Inserting (10), (11) and (12) into (8) and taking $y=N^{2}+\frac{1}{2}$, we obtain

$$
\sum_{f \in \mathcal{B}_{2}^{*}(N)} \lambda_{f}(n)=\frac{N}{12 \sqrt{n}} \delta_{n=\square}+O\left(N^{1-1 / 2 r} \tau(n) \mathcal{L}^{B_{6}}+N^{-1 / 2} x n^{1 / 2} \tau(n)\right),
$$

where we have used the fact that $\left(N x^{-1 / 2} n^{-1 / 4}+n^{-1 / 2}\right) \delta_{n=\square}$ can be absorbed by $N^{1-1 / 2 r} \tau(n)$ (since $\left.x^{r} \geq N^{10}\right)$. This completes the proof of Lemma 3 . 


\section{§3. Proof of the Theorem}

Now we are ready to prove our Theorem.

We start with the formula (7) instead of representing $L\left(\mathrm{sym}^{2} f, 1\right)$ by a sum of two absolutely convergent series as done in [1] (cf. 1], Lemma 4).

Let $r_{1} \geq 1$ be an integer and $x_{1}^{r_{1}} \geq N^{10}$. Define $r_{1}^{\prime}>1$ by $1 / r_{1}^{\prime}+1 / 2 r_{1}=1$. By the Hölder inequality, Lemma 2 and the fact that $\left|\mathcal{B}_{2}^{*}(N)\right| \asymp N$, there exists a positive constant $B_{7}=B_{7}\left(r_{1}\right)$ such that, for $y_{1}>x_{1}$ with $\log y_{1} \ll \mathcal{L}$,

$$
\begin{aligned}
\left|\sum_{f \in \mathcal{B}_{2}^{*}(N)} \omega_{f}\left(x_{1}, y_{1}\right)\right| & \leq\left|\mathcal{B}_{2}^{*}(N)\right|^{1 / r_{1}^{\prime}}\left(\sum_{f \in \mathcal{B}_{2}^{*}(N)} \omega_{f}\left(x_{1}, y_{1}\right)^{2 r_{1}}\right)^{1 / 2 r_{1}} \\
& \ll N^{1-1 / 2 r_{1}} \mathcal{L}^{B_{7}} .
\end{aligned}
$$

Thus we have

$$
\begin{aligned}
& \sum_{f \in \mathcal{B}_{2}^{*}(N)} L\left(\operatorname{sym}^{2} f, 1\right) \\
& \quad=\sum_{d^{2} m<x_{1}} \frac{\varepsilon_{N}(d)}{d^{2} m} \sum_{f \in \mathcal{B}_{2}^{*}(N)} \lambda_{f}\left(m^{2}\right)+O\left(N^{1-1 / 2 r_{1}} \mathcal{L}^{B_{7}}+N^{4} y_{1}^{-2}\right) .
\end{aligned}
$$

It remains to evaluate the last double sum. According to Lemma 3, we have

$$
\sum_{d^{2} m<x_{1}} \frac{\varepsilon_{N}(d)}{d^{2} m} \sum_{f \in \mathcal{B}_{2}^{*}(N)} \lambda_{f}\left(m^{2}\right)=\frac{N}{12} \sum_{d^{2} m<x_{1}} \frac{\varepsilon_{N}(d)}{d^{2} m^{2}}+R_{4},
$$

where

$$
\begin{aligned}
R_{4} & \ll N^{1-1 / 2 r} \mathcal{L}^{B_{6}} \sum_{d^{2} m<x_{1}} \frac{\tau\left(m^{2}\right)}{d^{2} m}+N^{-1 / 2} x \sum_{d^{2} m<x_{1}} \frac{\tau\left(m^{2}\right)}{d^{2}} \\
& \ll\left(N^{1-1 / 2 r}+N^{-1 / 2} x x_{1}\right) \mathcal{L}^{B_{8}},
\end{aligned}
$$

$x^{r} \geq N^{10}$ and $B_{8}=B_{8}(r)$ is a positive constant.

It is apparent that

$$
\begin{aligned}
\sum_{d^{2} m<x_{1}} \frac{\varepsilon_{N}(d)}{d^{2} m^{2}} & =\sum_{m<x_{1}} \frac{1}{m^{2}}\left\{\zeta_{N}(2)+O\left(\left(x_{1} / m\right)^{-1 / 2}\right)\right\} \\
& =\zeta_{N}(2) \sum_{m<x_{1}} \frac{1}{m^{2}}+O\left(x_{1}^{-1 / 2}\right) \\
& =\frac{\pi^{4}}{36}+O\left(x_{1}^{-1 / 2}+N^{-2}\right) .
\end{aligned}
$$

Combining these estimates yields

$$
\begin{aligned}
& \sum_{d^{2} m<x_{1}} \frac{\varepsilon_{N}(d)}{d^{2} m} \sum_{f \in \mathcal{B}_{2}^{*}(N)} \lambda_{f}\left(m^{2}\right) \\
& =\frac{\pi^{4}}{432} N+O\left(\left(N^{1-1 / 2 r}+N^{-1 / 2} x x_{1}+N x_{1}^{-1 / 2}\right) \mathcal{L}^{B_{8}}\right)
\end{aligned}
$$


Inserting (14) into (13), we find that

$$
\begin{aligned}
& \sum_{f \in \mathcal{B}_{2}^{*}(N)} L\left(1, \operatorname{sym}^{2} f\right) \\
& \quad=\frac{\pi^{4}}{432} N+O\left(\left(N^{1-1 / 2 r_{0}}+N^{-1 / 2} x x_{1}+N x_{1}^{-1 / 2}+N^{4} y_{1}^{-2}\right) \mathcal{L}^{B_{8}}\right),
\end{aligned}
$$

where $r_{0}:=\max \left\{r, r_{1}\right\}$. Now by taking $r=r_{1}=14, x=x_{1}=N^{41 / 56}$ and $y_{1}=N^{2}+\frac{1}{2}$, we obtain the desired result. This completes the proof of the Theorem.

\section{REFERENCES}

1. A. Akbary, Average values of symmetric square L-functions at $\Re$ es $=2$, C. R. Math. Rep. Acad. Sci. Canada 22 (3) (2000), 97-104. MR 2001h:11067

2. W. Duke, The critical order of vanishing of automorphic L-functions with large level, Invent. Math. 119 (1995), 165-174. MR 95k:11075

3. H. Iwaniec, W. Luo and P. Sarnak, Low lying zeros of families of L-functions, Inst. Hautes Études Sci. Publ. Math. 91 (2001), 55-131.

4. E. Kowalski and P. Michel, The analytic rank of $J_{0}(q)$ and zeros of automorphic L-functions, Duke Math. J. 100 (1999), 503-542. MR 2001b:11060

5. L. Mai and M. Ram Murty, The Phragmén-Lindelöf theorem and modular elliptic curves, Contemporary Math. 166 (1994), 335-340. MR 95g:11049

6. M. Ram Murty, The analytic rank of $J_{0}(N)(\mathbb{Q})$, CMS Conf. Proc. 15 (1995), 263-277. MR 96i:11054

Institut Élie Cartan, UMR 7502 UHP-CNRS-INRIA, Université Henri Poincaré (NANCY 1), 54506 VANdeUVRe-LÈs-NANCY, FranCE

E-mail address: wujie@iecn.u-nancy.fr 